\title{
Qualitative and Quantitative Biofilm Assay of Pseudomonas aeruginosa Isolates from Clinical Samples
}

\author{
Rekha Panwar ${ }^{1 *}$, Virendra Gehlot ${ }^{2}$, Ramkumar $^{1}$, Sunil Maherchandani $^{1}$ and $^{\text {Basant }}{ }^{1}$ \\ ${ }^{1}$ Department of Veterinary Microbiology and Biotechnology, University of Veterinary and \\ Animal Sciences, Bikaner (Rajasthan), India \\ ${ }^{2}$ Department of Dermatology and Venereology, Dr S N medical college, \\ Jodhpur (Rajasthan), India \\ *Corresponding author
}

\section{A B S T R A C T}

\begin{tabular}{|l|}
\hline Ke y w o r d s \\
$\begin{array}{l}\text { Pseudomonas } \\
\text { aeruginosa, } \\
\text { Biofilm, } \\
\text { Nosocomial } \\
\text { infection }\end{array}$ \\
\hline Article Info \\
\hline $\begin{array}{l}\text { Accepted: } \\
18 \text { May } 2020 \\
\text { Available Online: } \\
10 \text { June } 2020\end{array}$ \\
\hline
\end{tabular}

Pseudomonas aeruginosa is a Gram-negative bacterium found in multiple habitats, including soil, marine habitats and plants, can survive in harsh conditions such as high temperatures $42{ }^{\circ} \mathrm{C}$ and is capable of growing without oxygen. It is the fifth most commonly isolated organism and an opportunistic pathogen of animals. It is considered to be one of the major causes of nosocomial infections responsible for various clinical conditions such as pneumonia, urinary tract infections, and complications in clinical burns, wounds, and cystic fibrosis (CF). P. aeruginosa biofilm production is a well-known virulence factor and in the biofilm mode of growth it is involved in chronic as well as in acute infections. In present investigation 65 clinical sample of nosocomial infection taken from different animal species, namely dog, cattle camel, horse and buffalo. Total ten samples were found positive for Pseudomonas aeruginosa, which include four from Dog, one from Sheep, one from Goat, and four from Cattle whereas no samples were found positive for horse and buffalo. The aim of this study was to detect biofilm formation by study isolates of Pseudomonas aeruginosa qualitatively and quantitatively. Ten isolates were taken from diseased animal in veterinary clinics of RAJUVAS Bikaner. Qualitative biofilm formation assays (Pellicle formation assay and Congo red agar method) and quantitative assay by Microtitre plate assay used for biofilm production.

\section{Introduction}

Biofilms are highly-structured microbial communities that attach to different surfaces and are enclosed in a matrix of extracellular polymeric substance (EPS). Biofilms contain nucleic acids, protein, and other molecules (Mann and Wozniak 2012). These dynamic biofilms contain open water channels for transport of nutrients into the biofilm and waste products out of them (Stoodley et al., 2002). Bacterial biofilm consist of up to $97 \%$ of water, $2-5 \%$ of microbial cells and $3-6 \%$ of EPS and ions (Meliani and Bensoltane, 2015). Biofilms are formed by the bacteria in the environment and as well as during human and 
animal infections, whenever bacteria face any adverse condition. In $P$. aeruginosa biofuel production is a well-known virulence factor and in the biofilm mode of growth it is involved in chronic as well as in acute infections (Schaber et al., 2007). Pseudomonas organism can readily form biofilms as compared to other bacteria that require specific conditions such as temperature and $\mathrm{pH}$ (Clutterbuck, 2007). The process is initiated through secretion of matrix by $P$. aeruginosa cells and such cells bind to surfaces (Brown et al., 2012). This matrix provides a protective boundary that allows it to adhere to an environmental substrate. The coating of matrix confers 10- to 1000 -fold more protection to the organism against antibiotic treatment (Brown et al., 2012; Williams et al., 2015).

According to report of National Institute of Health, bacteria in biofilms are responsible for more than $60 \%$ of all bacterial infections (Bryers, 2008; Lewis, 2001). Bacteria in biofilms are more resistant to antimicrobial agents and host of immune defences as biofilms have barriers in the form of extracellular matrix and also there is presence of subpopulations of persisters that are physiological by less active and thus have high-level drug tolerance (Hall-Stoodley et al., 2004).

In $P$. aeruginosa, three exopolysaccharides (Psl, Pel and alginate) have been characterized for their role in the formation of biofilms (Kaplan, 2010; Wei and Ma, 2013). Alginate is the one of the most extensively characterized exopolysaccharides from mucoid strains, which forms highly viscous bacterial colonies on agar (Ramsey et al., 2005). It is responsible for providing nutrients an////d also helps in water retention in biofilms. It plays an important role in protection and structural stability of biofilms (Sutherland, 2001). Overproduction of alginate provides resistance to antibiotics also (Simpson et al., 1988). Taking view of the fact that, biofilms are responsible for the maximum resistance in $P$. aeruginosa.

\section{Materials and Methods}

\section{Isolation and species level conformation}

The preliminary isolation of ten Pseudomonas aeruginosa was carried out as per cultural characteristics and biochemical profiling from different clinical conditions (Cowan and Steel, 1974; Quinn et al., 1994).The clinical data regarding the distribution of isolates, type of specimens and type of infection are presented in table 1.

Proteomic based identification of Pseudomonas spp. by MALDI-TOF-MS (VITEK MS RUO)

Microbial identification with VITEK MS RUO (Research Use only) software and database was performed according to manufacturer's instructions by directly smearing pure and isolated colony from overnight cultured specimen (on blood agar) on to 48 positioned Flexi Mass DS disposable target plate (bioMerieux) and loaded with $1 \mu 1$ matrix (CHCA) solution. E. coli ATCC 8739 was used as the calibration strain. MALDITOF-MS analysis was performed in the linear positive mode in the mass range of $2-20 \mathrm{kDa}$.

\section{Maintenance of isolates}

The part of the pure culture stock was maintained on slants for short-term storage at $4^{\circ} \mathrm{C}$, while another part was stored in buffered glycerol $(1: 1)$ at $-80^{\circ} \mathrm{C}$.

\section{Characterization of biofilm production trait}

For the qualitative detection of biofilm all isolates grown on Congo Red Agar as per 
described by Freidman and Kolter (2004), then Pellicle formation at air-liquid interference assay was performed as per described by Chabane et al., (2014). For quantitative detection Microtiter plate assay was performed as per described previously by the method of O'Toole (2011).

\section{Qualitative biofilm assay}

\section{Slime production assay}

Slime production in $P$. aeruginosa strains were determined by cultivation on Congo red agar (CRA). Inoculated CRA plates were incubated at $37^{\circ} \mathrm{C}$ for $24 \mathrm{hr}$. The production of dark red and wrinkled colony morphology indicated the production of an extracellular matrix, which is involved in biofilm production.

\section{Pellicle formation assay}

A pellicle is a biofilm that assembles at the air-liquid interface of a standing liquid culture. Strains were inoculated in Luria Bertini broth supplemented with $1 \%$ glucose and incubated at $37^{\circ} \mathrm{C}$ for $24 \mathrm{hr}$. After incubation, a thin pellicle was formed at the air-liquid interface which is visible to the necked eye.

\section{Microtiter plate assay}

Biofilm production in microtiter plate was done as per the method of O'Toole, 2011.The bacterial culture was grown overnight at $37^{\circ} \mathrm{C}$ in $5 \mathrm{ml}$ Luria Bertini broth and overnight culture was then diluted with M63 medium. Two hundred $\mu 1$ of diluted cultures was added to each well of a 96 Microtiter plate. Each sample was inoculated in 3 wells in order to minimize errors. Six wells were used for negative control. The negative control wells contained only $200 \mu 1$ of M63 medium. The microtitre plate was incubated at $37^{\circ} \mathrm{C}$ for 24 hr. After incubation, the contents of the wells were decanted into a discard container and each well was washed three times with $300 \mu \mathrm{l}$ of sterile phosphate buffered saline. The plates were then drained in an inverted position and allowed to air dry at room temperature. Later $150 \mu \mathrm{l}$ of $0.1 \%$ crystal violet solution was added into wells and the excess stain was rinsed off by placing the microtiter plate under running tap water. The microtiter plate was allowed to air dry in an inverted position. After drying, the dye bound to the cells was resolubilized with $150 \mu 1$ of $33 \%$ glacial acetic acid. The plate was incubated at room temperature for 10-15 min. The optical density of each well stained with crystal violet was measured at $570 \mathrm{~nm}$ using a microtiter plate reader. The average OD values were calculated for all the tested strains and negative controls.

\section{Results and Discussion}

In the opportunistic pathogen Pseudomonas aeruginosa $(P$. aeruginosa $)$ biofilm is responsible for high level of drug resistance and requires attention (Chen et al., 2018). The ability to form biofilm is one of the main factors affecting pathogenicity of $P$. aeruginosa. All the isolates were tested for biofilm production phenotypically by three different methods viz., cultivation on modified Congo red agar, pellicle formation and microtiter plate assay.

Freeman et al., 1989used a simple qualitative method to detect biofilm production by using Congo Red Agar (CRA) medium. In case of $P$. aeruginosa production of dark red and wrinkled colonies indicated the production of an extracellular matrix, which is involved in biofilm formation (Freidman and Kolter, 2004).

In present study, all the ten $P$. aeruginosa isolates produced dark red and wrinkled 
colonies on modified Congo red agar, indicating the production of extracellular matrix.

$P$. aeruginosa is able to form biofilms not only on mucosal or other solid surfaces but also at the air-liquid interface of standing cultures and such structures are called as pellicles (Friedman and Kolter, 2004).Complete coverage of the surface of the culture by an opaque layer of cells and matrix material is considered as pellicle. In the present study all the ten isolates produced a pellicle at the air-liquid interface.
In the quantitative biofilm formation assay, results showed that all 10 isolates of Pseudomonas aeruginosa were produced biofilm strongly (OD was more than 0.25 ) in tissue culture plates when grown in M63 minimal medium. Biofilm production on tissue culture plates is considered the golden standard method for biofilm detection. The isolates were classified according to biofilm production according to the criteria laid down by Christensen as following: nonbiofilm producers less than 0.125 , weak biofilm producer between $0.125-0.25$ and strong biofilm producers more than 0.25 .

Table.1 Distribution of clinical isolates Pseudomonas spp. according to the type of specimens and infection

\begin{tabular}{|l|c|c|}
\hline $\begin{array}{l}\text { Study isolates no } \\
(\mathbf{\%})\end{array}$ & $\begin{array}{c}\text { Type of } \\
\text { infection }\end{array}$ & $\begin{array}{c}\text { Type of } \\
\text { specimen }\end{array}$ \\
\hline $\mathbf{2}(\mathbf{1 0 \%})$ & Otitis media & Ear swab \\
\hline $\mathbf{2 ( 1 3 . 3 3 \% )}$ & Burn & Wound swab \\
\hline $\mathbf{2 ( 2 0 \% )}$ & Surgical wound & Wound swab \\
\hline $\mathbf{1 ( 1 0 \% )}$ & Pus & Pus swab \\
\hline $\mathbf{3 ( 3 0 \% )}$ & UTI infection & Urine \\
\hline Total=10(15.38\%) & & \\
\hline
\end{tabular}

Bacterial communities in biofilms attach to surfaces, either living or abiotic and are surrounded by an extracellular matrix consisting of bacterium-derived DNA, proteins and exopolysaccharides. In nature, bacteria are considered to be mainly found in the form of biofilms. There is a realization that majority of the chronic or persistent infections are caused by bacteria in biofilms. In fact, 80 percent of medical bacterial infections are also caused by organisms growing in biofilms (Schaudinn et al., 2009). Bacteria in biofilm are highly resistant to immune system and antibiotics, however, most of the research on characterizing the physiology of bacteria and discovery of all current antibiotics and other antimicrobial products has been based on planktonic bacteria (Dosler and Karaaslan, 2014) forgetting the fact that established biofilms can be up to 1,000-fold more resistant to antibiotic treatment than planktonic bacteria, making them very resistant to current treatments (Lewis, 2001). In the opportunistic pathogen Pseudomonas aeruginosa (P. aeruginosa) biofilm is responsible for high level of drug resistance and requires attention (Chen et al., 2018).

Under the field of biofilm production, particularly qualitative biofilm assay by pellicle formation assay and congo red agar, the results showed that out of $10(15 \%)$ isolates of Pseudomonas aeruginosa formed biofilm as pellicle at air liquid interface. Further, in the other qualitative assay (congo 
red agar) our study revealed that out of 10 (15 $\%$ ) isolates of Pseudomonas aeruginosa, were produced red pigmented colony on congo red agar.

In order to enable easier study of bacterial attachment and colonization, a variety of experimental, direct and indirect, observation methods have been developed. Microtiter plate assay is the most frequently used techniques for quantifying biofilm formation Stepnovic et al., (2000). Microtiter plate procedure is an indirect method for estimation of bacteria in situ, it has the advantage of enabling researchers to rapidly analyze adhesion of multiple bacterial strains or growth conditions within each experiment, easy technique and used widely for antimicrobial agents susceptibility of biofilm.Our results showed that all pseudomonal isolates which were submitted to this study were produced biofilm stronglyat 570 nmoptical density

In conclusion, P.aeruginosa is an increasing global problem and a public health threat, posing many therapeutic challenges. Currently, it is the second most important opportunistic pathogen accounting for 10$11 \%$ of total nosocomial infections. Biofilm formation in $P$. aeruginosa infections is one of the main virulence determinants, which significantly increases bacterial resistance to antibiotics and innate host defenses.

Out of 65 samples processed a total of 10 isolates producing pyocynin were obtained on Cetrimide agar. These isolates were further tested preliminarily through Gram's staining and other primary biochemical tests and were confirmed as $P$. aeruginosa, as high confidence score value on MALDI TOF MS (VITEK MS RUO) was obtained.

All these 10 isolates were tested for biofilm production phenotypically by three different methods viz., cultivation on modified Congo red agar, pellicle formation and microtiter plate assay. All the $P$. aeruginosa isolates produced dark red and wrinkled colonies indicating the production of an extracellular matrix, which is involved in biofilm production. These isolates also produced pellicle at the air-liquid interface. All the isolates were able to produce biofilm in tissue culture plate assay.

\section{References}

Brown, A., Horobin, A., Blount, D. G., Hill, P. J., English, J., Rich, A., and Pritchard, D. I. (2012). Blow fly Lucilia sericata nuclease digests DNA associated with wound slough/eschar and with Pseudomonas aeruginosa biofilm. Medical and veterinary entomology, 26(4), 432-439.

Bryers, J.D., (2008). Medical biofilms. Biotechnology and bioengineering 100, 118.

Chabane, Y. N., Marti, S., Rihouey, C., Alexandre, S., Hardouin, J., Lesouhaitier, O., and Dé, E. (2014). Characterisation of pellicles formed by Acinetobacter baumannii at the air-liquid interface. PLoS One, 9(10), e111660.

Chen, H., Wubbolts, R. W., Haagsman, H. P., and Veldhuizen, E. J. (2018). Inhibition and Eradication of Pseudomonas aeruginosa Biofilms by Host Defence Peptides. Scientific reports, 8(1), 10446.

Christensen, B. B., Sternberg, C., Andersen, J. B., Eberl, L., Møller, S., Givskov, M., and Molin, S. (1998). Establishment of new genetic traits in a microbial biofilm community. Appl. Environ. Microbiol., 64(6), 2247-2255.

Clutterbuck, A. L., Woods, E. J., Knottenbelt, D. C., Clegg, P. D., Cochrane, C. A., and Percival, S. L. (2007). Biofilms and their relevance to veterinary medicine. Veterinary microbiology, 121(1-2), 1-17.

Cowan, S. T., and Steel, K. J. (1974). Enterobacteriaceae. Bergey's Manual of Determinative Bacteriology, 8th ed. Eds RE Buchanan and NE Gibbons. The Williams and Wilkins Co., Baltimore, 290-293. 
Dosler, S., and Karaaslan, E. (2014). Inhibition and destruction of Pseudomonas aeruginosa biofilms by antibiotics and antimicrobial peptides. Peptides, 62, 32-37.

Freeman, D. J., Falkiner, F. R., and Keane, C. T. (1989). New method for detecting slime production by coagulase negative staphylococci. Journal of clinical pathology, 42(8), 872-874.

Friedman, L., and Kolter, R. (2004). Two genetic loci produce distinct carbohydrate-rich structural components of the Pseudomonas aeruginosa biofilm matrix. Journal of bacteriology, 186(14), 4457-4465.

Hall-Stoodley, L., Costerton, J.W., Stoodley, P., (2004). Bacterial biofilms: from the natural environment to infectious diseases. Nature reviews microbiology 2, 95 .

Kaplan, J. Á. (2010). Biofilm dispersal: mechanisms, clinical implications, and potential therapeutic uses. Journal of dental research, 89(3), 205-218.

Lewis, K., (2001). Riddle of biofilm resistance. Antimicrobial agents and chemotherapy 45, 999-1007.

Mann, E. E., and Wozniak, D. J. (2012). Pseudomonas biofilm matrix composition and niche biology. FEMS microbiology reviews, 36(4), 893-916.

Meliani, A., and Bensoltane, A. (2015). Review of Pseudomonas attachment and biofilm formation in food industry. Poultry, Fisheries and Wildlife Sciences, Pp. 1-7.

O'Toole, G.A., (2011). Microtiter dish biofilm formation assay. Journal of visualized experiments: JoVE.

Quinn, P., Carter, M., Markey, B., Carter, G., (1994). Veterinary clinical microbiology. Wolfe Publication, London, UK, 254-258.

Ramsey, D. M., and Wozniak, D. J. (2005). Understanding the control of Pseudomonas aeruginosa alginate synthesis and the prospects for management of chronic infections in cystic fibrosis. Molecular microbiology, 56(2), 309-322.

Schaber, J. A., Triffo, W. J., Suh, S. J., Oliver, J. W., Hastert, M. C., Griswold, J. A., and Rumbaugh, K. P. (2007). Pseudomonas aeruginosa forms biofilms in acute infection independent of cell-to-cell signaling. Infection and immunity, 75(8), 3715-3721.

Schaudinn, C., Gorur, A., Keller, D., Sedghizadeh, P. P., and Costerton, J. W. (2009). Periodontitis: an archetypical biofilm disease. The Journal of the American Dental Association, 140(8), 978986.

Simpson, J. A., Smith, S. E., and Dean, R. T. (1988). Alginate inhibition of the uptake of Pseudomonas aeruginosa by macrophages. Microbiology, 134(1), 29-36.

Stepanović, S., Ćirković, I., Ranin, L., and Svabić-Vlahović, M. (2004). Biofilm formation by Salmonella spp. and Listeria monocytogenes on plastic surface. Letters in applied microbiology, 38(5), 428-432.

Stoodley, P., Sauer, K., Davies, D.G., Costerton, J.W., (2002). Biofilms as complex differentiated communities. Annu Rev Microbiol 56, 187-209.

Sutherland, I. W. (2001). Biofilm exopolysaccharides: a strong and sticky framework. Microbiology, 147(1), 3-9.

Wei, Q., and Ma, L. Z. (2013). Biofilm matrix and its regulation in Pseudomonas aeruginosa. International journal of molecular sciences, 14(10), 20983-21005.

Williams, D. L., Vinciguerra, J., Lerdahl, J. M., and Bloebaum, R. D. (2015). Does vitamin E-blended UHMWPE prevent biofilm formation? Clinical Orthopaedics and Related Research®, 473(3), 928-935.

\section{How to cite this article:}

Rekha Panwar, Virendra Gehlot, Ramkumar, Sunil Maherchandani and Basant. 2020. Qualitative and Quantitative Biofilm Assay of Pseudomonas aeruginosa Isolates from Clinical Samples. Int.J.Curr.Microbiol.App.Sci. 9(06): 1708-1713.

doi: https://doi.org/10.20546/ijcmas.2020.906.211 